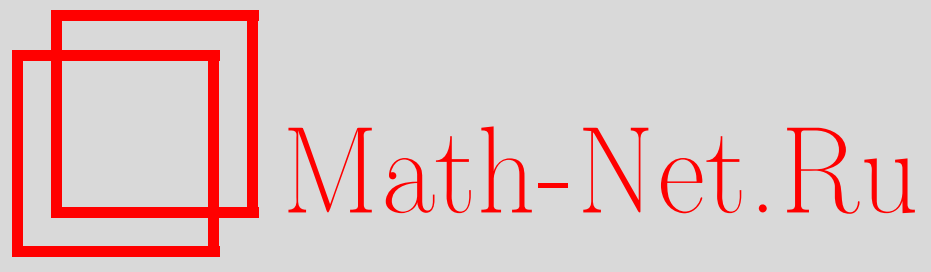

Е. Е. Скурихин, Об одном классе категорных топологических пространств, УМH, 2008, том 63, выпуск 1, 167168

DOI: https://doi.org/10.4213/rm9160

Использование Общероссийского математического портала Math-Net.Ru подразумевает, что вы прочитали и согласны с пользовательским соглашением http://www . mathnet.ru/rus/agreement

Параметры загрузки:

IP: 3.82 .47 .9

26 апреля 2023 г., 14:04:48

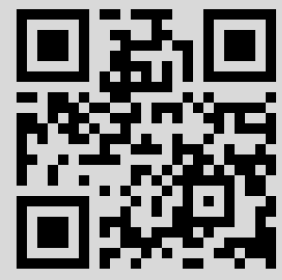




\section{Об одном классе категорных топологических пространств}

\section{Е. Е. Скурихин}

Категорное топологическое пространство - это обобщенный объект произвольного сайта Гротендика вместе со структурой его подобъектов [1], [2]. Результаты работы [3] мы применяем к случаю, когда сайт является нижней полурешеткой, а топология Гротендика задается единственным семейством. Определяются длины Чу-пространства и устанавливаются их связи с когомологическими размерностями, в том числе с вялой. Дается когомологическая характеристика размерности нётерова пространства и значит алгебраического многообразия.

Пусть $K-\wedge$-полурешетка, $\alpha=\left\{a_{i} \in K \mid i \in I\right\}$ - семейство элементов, $\tau$ топология Гротендика на $K$. Если $K$ имеет нуль 0, то топология Гротендика $\tau^{\prime}$ определяется так: $\tau^{\prime}(a)=\tau(a)$, если $a \neq 0$, и $\tau^{\prime}(0)=\tau(0) \cup\{\varnothing\}$. Определим для всякого $a \in K$ класс семейств $\tau_{\alpha}(a)$, полагая для $\beta=\left\{b_{j} \in K \mid j \in J\right\}: \beta \in \tau_{\alpha}(a)$ тогда и только тогда, когда $\beta \in \tau(a)$ и $\alpha \wedge a \prec \beta$. Легко видеть, что $\tau_{\alpha}$ - топология Гротендика на $K$. Будем в индексах писать $\mu$ вместо $\tau_{\alpha}^{\prime}$.

Зафиксируем $\gamma=\left\{c_{l} \in K \mid l \in L\right\}$ и введем следующие обозначения: $K_{1}-$ множество всех подпредпучков предпучка множеств 1 такого, что $1(a)$ - одноэлементное множество для любого $a \in K ; \mathscr{U}=\left\{\gamma_{M} \wedge a \mid M \subset K, a \in K\right\}$, где $\gamma_{M}=\left\{c_{l} \in K \mid l \in M\right\}$; если $\delta$ - семейство элементов $K$, то $\tilde{\delta} \in K_{1}$ - предпучок множеств, для которого $\delta$ является $\mu$-покрытием; $\widetilde{\mathscr{U}}=\{\tilde{\delta} \mid \delta \in \mathscr{U}\}$.

Теорема 1. Пусть $\delta$-семейство элементов $K, \delta \prec \alpha$.

1) Если $\mathscr{A}$ - абелев предпучок и $\mathscr{A}(0)=0$, то для любого $n \geqslant 0 \check{H}_{\mu}^{n}(\tilde{\delta}, \mathscr{A})=$ $H_{\mu}^{n}\left(\tilde{\delta}, S_{\mu}(\mathscr{A})\right)=H^{n}(\delta, \mathscr{A})=\lim ^{(n)}\left(\mathscr{A} \mid R_{\delta}\right)$, где справа стоит $n-\check{u}$ производный функтор обратного предела ограничения абелева предпучка $\mathscr{A}$ на решето $R_{\delta} \subset K$, порожденное семейством $\delta$. Здесъ $S_{\mu}(\mathscr{A})$ обозначает $\mu$-пучок, порожденный $\mathscr{A}, \check{H}_{\mu}^{n}$ и $H_{\mu}^{n}$ - когомологии Чеха и Гротендика соответственно.

2) Если кратность $\delta$ не превышает $k$, то для любого $\mu$-пучка $\mathscr{A} H_{\mu}^{m}(\tilde{\delta}, \mathscr{A})=0$ при $m \geqslant k$.

Назовем $\mu$-пучок $\mathscr{A} \mathscr{U}$-вялым, если для любого $\delta \in \mathscr{U}$ отображение $H^{0}(\gamma, \mathscr{A}) \rightarrow$ $H^{0}(\delta, \mathscr{A})$ является эпиморфизмом.

Tеорема 2. Пусть $\gamma \prec \alpha$.

1) Если $\mathscr{A}$ есть $\mathscr{U}$-вялый $\mu$-пучок, $C=\tilde{\gamma}$, то для любого $B \in \tilde{\mathscr{U}}$ и любого $n \geqslant 1$ $H_{\mu}^{n}(C, \mathscr{A})=H_{\mu}^{n}(B, \mathscr{A})=H_{\mu}^{n}(C, B, \mathscr{A})=0$.

2) Если кратность $\gamma$ не превышает $n+1$, то любой абелев $\mu$-пучок имеет вялую резольвенту длины $\leqslant n+1$.

Тройка $\mathscr{X}=(X, Y, r: X \times Y \rightarrow\{0,1\})$, где $X, Y$ - множества и $r$ - отображение, называется Чу-пространством [4], [5]. Определим множества $X_{y} \subset X$ и $Y_{x} \subset Y$, полагая $x \in X_{y} \Leftrightarrow y \in Y_{x} \Leftrightarrow r(x, y)=1$. Длиной семейства $\left\{x_{i} \in X \mid i \in I\right\}$ назовем супремум по $y \in Y$ мощностей множеств $\left\{i \in I \mid x_{i} \in X_{y}\right\}$. Симметрично определяется длина семейства элементов $Y$. Левой длиной $\mathrm{l} L \mathscr{X}$ назовем длину $X$, а правой $\mathrm{rL} \mathscr{X}-$ длину $Y$. Через $\tau$ будем обозначать канонические топологии Гротендика на упорядоченных по включению множествах $S(X)$ и $S(Y)$ всех подмножеств соответственно $X$ и $Y$. Это означает, что семейство множеств является $\tau$-покрытием некоторого множества $A$ тогда и только тогда, когда его объединение равно $A$. Пусть $\alpha=\left\{X_{y} \mid y \in Y\right\}$, $\beta=\left\{Y_{x} \mid x \in X\right\}$. Обозначим: $\tau_{1}=\tau_{\alpha}^{\prime}, \tau_{\mathrm{r}}=\tau_{\beta}^{\prime}$ - соответственно левая и правая топологии на $\mathscr{X}, H_{1}^{n}(\mathscr{X}, \mathscr{A})=H_{\mu}^{n}(X, \mathscr{A}), H_{\mathrm{r}}^{n}(\mathscr{X}, \mathscr{A})=H_{\mu}^{n}(Y, \mathscr{B})$ - соответственно левые и правые когомологии Гротендика $\mathscr{X}$ с коэффициентами в $\mu$-пучке $\mathscr{A}$, соответственно $\mathscr{B}$, где $\mu=\tau_{\alpha}^{\prime}$, соответственно $\mu=\tau_{\beta}^{\prime}$.

Работа выполнена при поддержке грантов ДВО РАН 06-III-A-01-015 и НШ-9004.2006.1. 
Отметим, что длина семейства $\left\{x_{i} \in X \mid i \in I\right\}$ равна кратности семейства $\left\{Y_{x_{i}} \subset\right.$ $Y \mid i \in I\}$. Поэтому следующий результат получается из сформулированных выше общих результатов.

Если $\gamma$ - семейство подмножеств $X$, то через $\gamma_{X}$ обозначаем семейство, получаемое из $\gamma$ добавлением всех одноточечных подмножеств $X$, не входящих в объединение $\gamma$.

Teорема 3. Пусть $\mathscr{X}=(X, Y, r: X \times Y \rightarrow\{0,1\})-$ Чу-пространство.

1) Если $\mathscr{A}$ - абелев предпучок на $S(X), \mathscr{A}(\varnothing)=0$ и $S_{l}(\mathscr{A})$ обозначает $\tau_{1}-$ пучок, порожденный $\mathscr{A}$, то для любого $n \geqslant 0 H_{l}^{n}\left(\mathscr{X}, S_{l}(\mathscr{A})\right)=H^{n}\left(\alpha_{X}, \mathscr{A}\right)$.

2) Если $\mathrm{lL} \mathscr{X} \leqslant n$ и $\mathscr{B}-\tau_{\mathrm{r}}$-пучок, то для любого $k>n H_{r}^{k}(\mathscr{X}, \mathscr{B})=0$ и $\mathscr{B}$ имеет $r$-вялую резольвенту длинь $\leqslant n$. Здесъ $r$-вялость означает $\mathscr{U}$-вялость, где $\mathscr{U}$ строится по семейству $\gamma=\beta$.

Teоpema 4. Пусть $\mathscr{X}=(X, \mathscr{M}, r: X \times \mathscr{M} \rightarrow\{0,1\})-$ Чy-nространство, где $X-$ множество, $\mathscr{M}$ - некоторое множество его непустых подмножеств, отображение $r$ задается равенством $r(x, A)=1 \Leftrightarrow x \in A$. Тогда lL $\mathscr{X}$ равняется супремуму мощностей элементов $\mathscr{M}$. Предположим, что условия $A \in \mathscr{M} u B \subset A$ влекут $B \in \mathscr{M}$. Тогда если $\mathrm{IL} \mathscr{X}=n$, то имеется абелев $\tau_{\mathrm{r}}$-пучок $\mathscr{A}$ такой, что $H_{\mathrm{r}}^{n}(\mathscr{X}, \mathscr{A}) \neq 0$.

Теорема 5. Пусть $X$ - нётерово топологическое пространство, $\mathscr{X}=(I(X)$, $\mathscr{M}, r: I(X) \times \mathscr{M} \rightarrow\{0,1\})$ - Чу-пространство, где $I(X)$ - множество всех замкнутых неприводимых подмножеств $X, \mathscr{M}$ - множество линейно упорядоченных по включению подмножеств $I(X), r(A, M)=1 \Leftrightarrow A \in M$. Тогда размерность $X$ равна правой когомологической размерности $\mathscr{X}$, т.е. максимальному п такому, что $H_{\mathrm{r}}^{n}(\mathscr{X}, \mathscr{A}) \neq 0$ для некоторого $\tau_{\mathrm{r}}$-пучка $\mathscr{A}$. В частности, это так, если $X$ - алгебрачческое многообразие.

Пусть $\mathscr{X}=(X, Y, r: X \times Y \rightarrow\{0,1\})$ и $\mathscr{X}^{\prime}=\left(X^{\prime}, Y^{\prime}, r^{\prime}: X^{\prime} \times Y^{\prime} \rightarrow\{0,1\}\right)-$ Чу-пространства. Морфизмом $\mathscr{X}$ в $\mathscr{X}^{\prime}$ называется пара отображений $\left(f: X \rightarrow X^{\prime}\right.$, $\left.g: Y^{\prime} \rightarrow Y\right)$ такая, что для любых $x$ и $y^{\prime} r^{\prime}\left(f(x), y^{\prime}\right)=r\left(x, g\left(y^{\prime}\right)\right)$.

Теорема 6. Пусть $(f, g)$ - морфизм Чу-пространств, $\alpha^{\prime}=\left\{X_{y^{\prime}}^{\prime} \mid y^{\prime} \in Y^{\prime}\right\}, \beta=$ $\left\{Y_{x} \mid x \in X\right\}$.

1) Для любого абелева предпучка $\mathscr{A}$ на $S(X)$ такого, что $\mathscr{A}(\varnothing)=0$, существует спектральная последовательность со вторым членом $E_{2}^{p q}=H_{1}^{p}\left(\mathscr{X}^{\prime}, \mathscr{E}_{f}^{q}(\mathscr{A})\right)$, сходящаяся $\kappa H^{*}\left(f^{-1}\left(\alpha^{\prime}\right)_{X}, \mathscr{A}\right)$, где предпучок $\mathscr{E}_{f}^{q}(\mathscr{A})$ на $S\left(X^{\prime}\right)$ задается равенством

$$
\mathscr{E}_{f}^{q}(\mathscr{A})(C)=H^{q}\left(f^{-1}\left(\alpha^{\prime}\right)_{X} \wedge f^{-1}(C), \mathscr{A}\right)=H^{q}\left(f^{-1}\left(\alpha^{\prime}\right)_{X} \wedge f^{-1}(C), S_{l}(\mathscr{A})\right) .
$$

2) Для любого абелева предпучка $\mathscr{B}$ на $S\left(Y^{\prime}\right)$ такого, что $\mathscr{B}(\varnothing)=0$, существует спектральная последовательность со вторым членом $E_{2}^{p q}=H_{\mathrm{r}}^{p}\left(\mathscr{X}, \mathscr{E}_{g}^{q}(\mathscr{B})\right)$, сходящаяся $\kappa H^{*}\left(g^{-1}(\beta)_{Y}^{\prime}, \mathscr{B}\right)$, где предпучок $\mathscr{E}_{g}^{q}(\mathscr{B})$ на $S(Y)$ задается равенством

$$
\mathscr{E}_{g}^{q}(\mathscr{B})(C)=H^{q}\left(g^{-1}(\beta)_{Y}^{\prime} \wedge g^{-1}(C), \mathscr{B}\right)=H^{q}\left(g^{-1}(\beta)_{Y}^{\prime} \wedge g^{-1}(C), S_{\mathrm{r}}(\mathscr{B})\right) .
$$

\section{Список литературы}

[1] Е. Е. Скурихин, Топология и ее приложения, Тр. междунар. топологической конф. (Баку, 1987), Тр. МИАН, 193, Наука, М., 1992, 169-173. [2] Е. Е. Скурихин, Дискретнал геометрия и геометрия чисел, Тр. МИАН, 239, Наука, М., 2002, 289-317. [3] Е. Е. Скурихин, Пучки на нормальных и паракомпактных решетках, Дальнаука, Владивосток, 1998. [4] Barr M., *-autonomous categories, Lecture Notes in Math., 752, Springer, Berlin, 1979. [5] Е. Е. Скурихин, А. Г. Сухонос, Дальневост. мат. журн., 6:1-2 (2005), 14-22.

Е. Е. Скурихин (E. E. Skurikhin)

Институт прикладной математики ДВО РАН

E-mail: eesku@iam.dvo.ru
Представлено В. М. Бухштабером Принято редколлегией 29.12 .2007 